\title{
Tunable, narrow-band, grating-assisted microring reflectors
}

\author{
Carmen Vázquez ${ }^{\mathrm{a}}$, Otto Schwelb ${ }^{\mathrm{b}, *}$
}

a Departamento Tecnología Electrónica, Universidad Carlos III, Leganés 28911, Madrid, Spain

${ }^{\mathrm{b}}$ Department of Electrical and Computer Engineering, Concordia University, Montréal, QC, Canada H3G 1 M8

A R T I C L E I N F O

Article history:

Received 26 March 2008

Received in revised form 20 June 2008

\begin{tabular}{l}
\hline Keywords: \\
Microring resonator \\
Laser mirror \\
Optical reflector \\
Group delay \\
\hline
\end{tabular}

Accepted 21 June 2008

\section{A B S T R A C T}

Tunable, grating-assisted ring resonator based optical mirrors are analyzed and their characteristics numerically evaluated. Mode number selection rules are presented to aid mirror optimization for high, narrow-band reflection and efficient sidelobe suppression. Data is presented on tuning sensitivity, on the effect of coupling on bandwidth and reflection coefficient, and on the influence of waveguide and grating loss on mirror performance. Group delay in the presence of loss and circuit parameter variations is also treated.

\section{Introduction}

Microring resonator based optical mirrors and band-limited reflectors have been the subject of intense investigations in recent years, primarily as a result of advancements in fabrication technology and miniaturization of integrated devices [1-6]. In this paper we present analytical and simulated results on the performance of three closely related narrow-band laser mirror configurations which use Bragg gratings to confine their operating range. The gratings provide the means to suppress repetition of the reflection spectrum at every free spectral range (FSR) removed from the design wavelength, and permit a laser with a wide gain characteristic to oscillate at several frequencies. The device consists of a single microring resonator and two identical Bragg gratings as shown in Fig. 1. It can be viewed as a phase shifted Fabry-Pérot resonator that uses gratings as mirrors and a coupling mechanism to transfer its spectrum to the laser that includes a microring. The difference between the configurations shown in Fig. 1 is in the method the gratings are attached to the microring. One of the configurations has been discussed in a different context in [7-10] while another was briefly treated in [11]. Although the three variants appear to be similar, their characteristics and fabrication differ in respects that determine the choice of application. For example, the type I mirror of Fig. 1 indicates geometry where the connection between the resonator and the Bragg gratings might require very small bending radii and consequently precision waveguide fabrication to avoid exces-

* Corresponding author. Tel.: +1 514573 9155; fax: +1 5148482802 .

E mail addresses: cvazquez@ing.uc3m.es (C. Vázquez), otto@ece.concordia.ca (O. Schwelb). sive radiation loss. Resolution of this problem is offered in the type III mirror configuration which, however, requires vertically displaced waveguides and the associated more critical fabrication technology. Tunability, bandwidth (BW), sidelobe suppression (SS), sensitivity to loss and the most convenient placement of (thermo-optic) tuning pads are other aspects differentiating the three configurations.

In Section 2 we present a network analysis of the reflectors and plot the spectral characteristics of the reflected intensity. Here we also discuss the selection rules that determine the optimum mode numbers of the ring and the grating arms. Tuning sensitivity is treated in Section 3 while in Section 4 BW and the effects of waveguide and grating loss on reflectivity are presented. Section 5 is devoted to computed results on group delay in reflection [12] and in Section 6 we draw some conclusions.

\section{Analysis and spectral characteristics}

The common circuitry of the reflector configurations are depicted schematically at the top part of Fig. 1, showing the bus waveguide with input and output ports, the microring with circumference $L$, the gratings, and the two couplers $K_{\mathrm{c}}$ and $K$. At the bottom of this figure are shown, for the three reflector configurations, the direct (solid lines) and coupled (dotted lines) connections within the coupler denoted by $K$. Accordingly, in the type I mirror the ring section $(1-h) L$ is directly connected to arm $l_{3}$ and $h L$ to $l_{4}$, whereas in the type III mirror the reverse is true. In the type II mirror one of the direct connections closes the ring while the other connects the two gratings, thus when the coupling is reduced to zero the gratings are completely separated from the ring. 

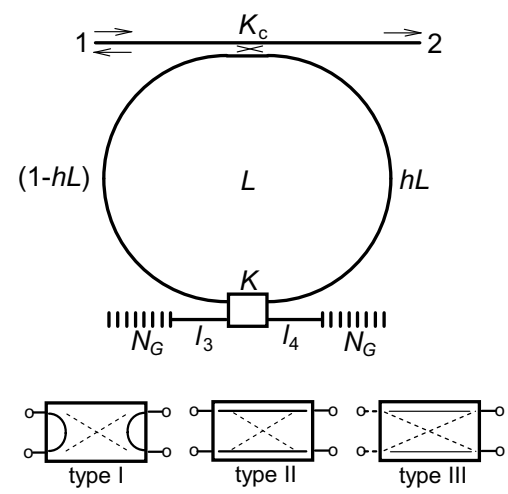

Fig. 1. Three types of grating-assisted microring resonator mirrors. The direct and coupled connections of the coupler $K$, detailed at the bottom, are indicated by the solid and dotted lines, respectively. The ring perimeter is $L, N_{G}$ is the number of periods in each grating, and $h(0<h<1)$ locates the coupler $K$ on the ring. The attenuation coefficient of the waveguides is $\alpha$, the ring need not be circular.

$K$ represents the power coupling coefficient of the bottom cou pler, and $K_{\mathrm{c}}$ that of the coupler between the ring and the bus wave guide. The location of $K$ within the ring, determined by $h(0<h<1)$, is arbitrary. We assume that all four waveguide sections, namely $h L$, ( $1 h) L, l_{3}$ and $l_{4}$, are characterized by the same effective refractive index $n_{\text {eff }}$ and the same amplitude attenuation coefficient $\alpha$, although these restrictions can easily be relaxed. We also admit localized or distributed gain in the ring [13 15 ] and thermo optic length or refractive index adjustment $[16,17]$ for $L, l_{3}, l_{4}$ and $n_{\text {eff- }}$ All optical lengths are given in terms of mode numbers, thus $N$ $L n_{\text {eff }} / \lambda_{0}$ and $n_{i} \quad l_{i} n_{\text {eff }} / \lambda_{0}, i \quad 3,4$, where $\lambda_{0} \quad c / f_{0}$ is the design wave length and $c$ the velocity of light in vacuum. The gratings are identi cal, quarter wavelength shallow gratings, meaning that the relative index difference $\left(n_{b} \quad n_{a}\right) / n_{a} \ll 1,\left(n_{b}>n_{a}\right)$. The gratings are charac terized by the grating strength $G S \quad N_{G}\left(n_{b} \quad n_{a}\right) / n_{a}$ where $N_{G}$ is the number of periods. Balanced operation is ensured by selecting $K \cong 0.5$, while $K_{\mathrm{c}}$ must be small to prevent loading and to preserve the resonance spectrum of the grating assisted ring [18]. Here we remark that the type II mirror of Ref. [11], using $K_{\mathrm{c}} 0.5$ and $0.01 \leqslant K \leqslant 0.05$ also produces a very high SS, relatively low reflec tion loss (RL) mirror. Coupling scheme differences result in signifi cant diversity in performance as well as demands on fabrication. Foremost among these is the extremely large SS and the heightened sensitivity to $K$ in the vicinity of $K \quad 0.5$, observed in type I mirrors for a particular mode number combination, as described below.

To simplify the analysis we combine the coupler $K$ and the two gratings, including their arms $l_{3}$ and $l_{4}$, into a single two port net work characterized by its scattering matrix $\boldsymbol{S}_{K}$ which can be calcu lated from first principles and is found to be

$\boldsymbol{S}_{K} \quad\left[\begin{array}{lll}c^{2} \Gamma_{3} & s^{2} \Gamma_{4} & \mathrm{j} s c\left(\Gamma_{3}+\Gamma_{4}\right) \\ \mathrm{j} s c\left(\Gamma_{3}+\Gamma_{4}\right) & c^{2} \Gamma_{4} & s^{2} \Gamma_{3}\end{array}\right]$

for the type I configuration,

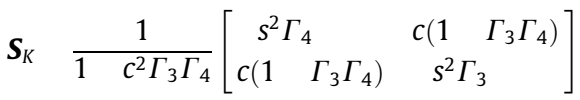

for the type II configuration, and

$$
\mathbf{S}_{K} \frac{1}{1+s^{2} \Gamma_{3} \Gamma_{4}}\left[\begin{array}{ll}
c^{2} \Gamma_{4} & \mathrm{j} s\left(1+\Gamma_{3} \Gamma_{4}\right) \\
\mathrm{j} s\left(1+\Gamma_{3} \Gamma_{4}\right) & c^{2} \Gamma_{3}
\end{array}\right]
$$

for the type III configuration, where $c(1 K)^{1 / 2}, s \quad(K)^{1 / 2}$, and

$$
\Gamma_{i} \frac{\mathrm{j} \kappa^{*} \operatorname{sh}\left(\Gamma_{G} N_{G, i} \Lambda\right)}{\gamma_{G} \operatorname{ch}\left(\gamma_{G} N_{G, i} \Lambda\right)+(\alpha+\mathrm{j} \Delta \beta) \operatorname{sh}\left(\gamma_{G} N_{g, i} \Lambda\right)} \exp \left[2(\alpha+\mathrm{j} \beta) l_{i}\right]
$$

$i$ 3, 4 are the complex reflection coefficients of arms 3 and 4, con sisting of guide lengths $l_{3}$ and $l_{4}$, respectively, terminated by their corresponding gratings of lengths $N_{G, i} \Lambda$, where $\Lambda$ is the periodicity, $\Delta \beta \quad \beta \quad \pi / \Lambda$ is the detuning, $\alpha$ is the amplitude attenuation in the waveguides, $\gamma_{G}\left[|\kappa|^{2}+(\alpha+\mathrm{j} \Delta \beta)^{2}\right]^{1 / 2}$ and $\kappa$ is the coefficient that measures the coupling between forward and backward traveling waves in the grating [19].

The relevant elements of the scattering matrix $\boldsymbol{S}$ of the entire reflector are the reflection coefficient $S_{11}$ and the transmission coefficient $S_{21}$, both complex valued functions of frequency. Assuming $\alpha \quad 0$ and $h \quad 0.5$, tedious but straightforward algebra yields

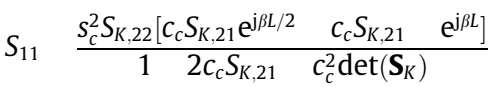

and

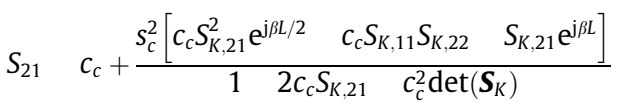

where $c_{\mathrm{c}}\left(1 K_{\mathrm{c}}\right)^{1 / 2}, s_{\mathrm{c}}\left(K_{\mathrm{c}}\right)^{1 / 2}$ and $\operatorname{det}\left(\mathbf{S}_{K}\right) \Gamma_{3} \Gamma_{4},\left(\begin{array}{lll}\Gamma_{3} \Gamma_{4} & \left.c^{2}\right) /\end{array}\right.$ (1 $\left.c^{2} \Gamma_{3} \Gamma_{4}\right)$ or $\left(\Gamma_{3} \Gamma_{4}+s^{2}\right) /\left(1+s^{2} \Gamma_{3} \Gamma_{4}\right)$, for mirror types I, II or III, respectively. When dealing with lossy waveguides $\beta$ must be re placed by $\beta$ j $\alpha$. When a semiconductor optical amplifier (SOA) is embedded in the ring to compensate losses, a negative $\alpha$ can be used such that its product with the perimeter length $L$ equals the gain of the SOA.

Denoting the reflected and transmitted intensity of the reflec tors by $I_{11} \quad\left|S_{11}\right|^{2}$ and $I_{21} \quad\left|S_{21}\right|^{2}$, respectively, the computed spec tral response of the lossless devices are plotted in Figs. 24 for three values of the resonator mode number $N$, as a function of detuning from the design frequency $f_{0}$, the center frequency of the grating passband. In our calculations we used $K_{\mathrm{c}} 0.02, \mathrm{~K}$ $0.4944, N_{G} 600$ (GS 3.92), a center wavelength of $\lambda_{0} 1.579$ $\mu \mathrm{m}$ and $n_{\text {eff }} \quad 4.5$, appropriate for SOI waveguides [20].

There are two kinds of type I mirrors, namely the very large SS variety whose spectrum is shown in Fig. 2 and the moderate SS variety whose spectrum appears in Fig. 3. They differ in the mode number of one of the grating arms, i.e., in the relative reflection phase of the grating reflectors. The difference is $180^{\circ}$, so for

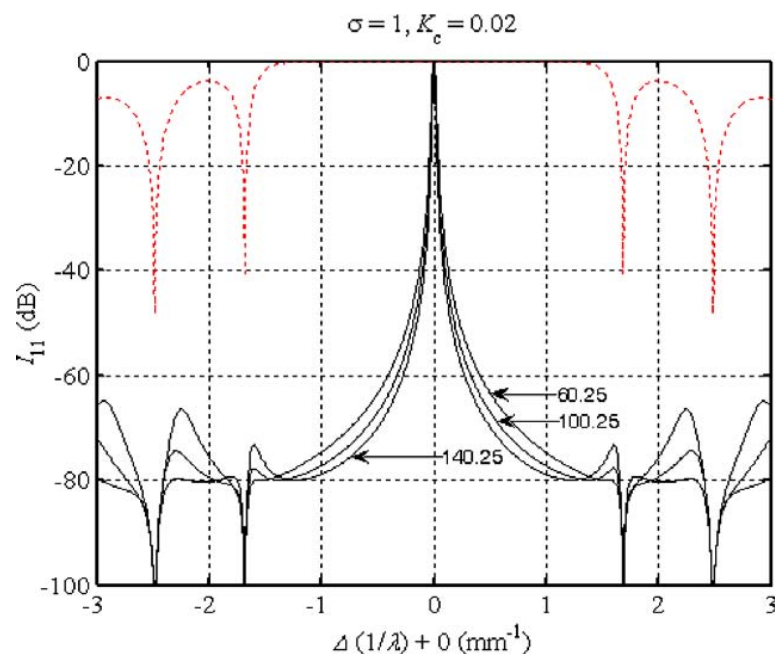

Fig. 2. Reflected intensity of the type I mirror for three mode numbers when $n_{3}=n_{4}=10.25$. Also shown (dotted line) is the reflection loss characteristics of the gratings. $N_{G}=600, n_{a}=4.5294, n_{b}=n_{\text {eff }}=4.5 \quad(G S=3.92), K=0.4944, \quad K_{\mathrm{c}}=0.02$, $\lambda_{0}=1.5791 \mu \mathrm{m}$ and $\sigma=\exp (\alpha L)$. Multiply the abscissa by $3 \times 10^{2}$ to obtain the detuning in $\mathrm{GHz}$. As $|K-0.5| \rightarrow 0$ the SS increases. 


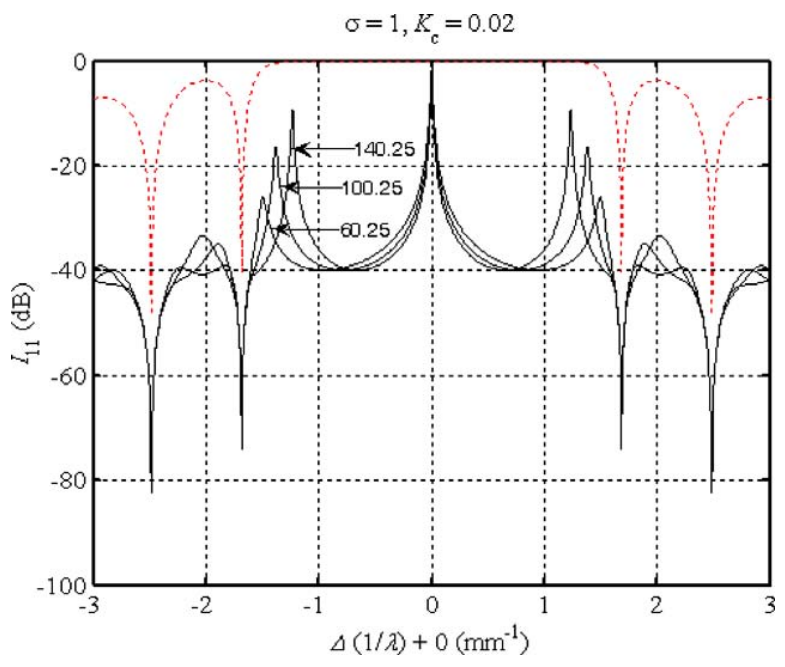

Fig. 3. Reflected intensity of the type I mirror for three mode numbers when $n_{3}=10.25$ and $n_{4}=10$. All other parameters are the same as in Fig. 2. Notice the reduced SS, virtually independent of $K$, in comparison to Fig. 2.

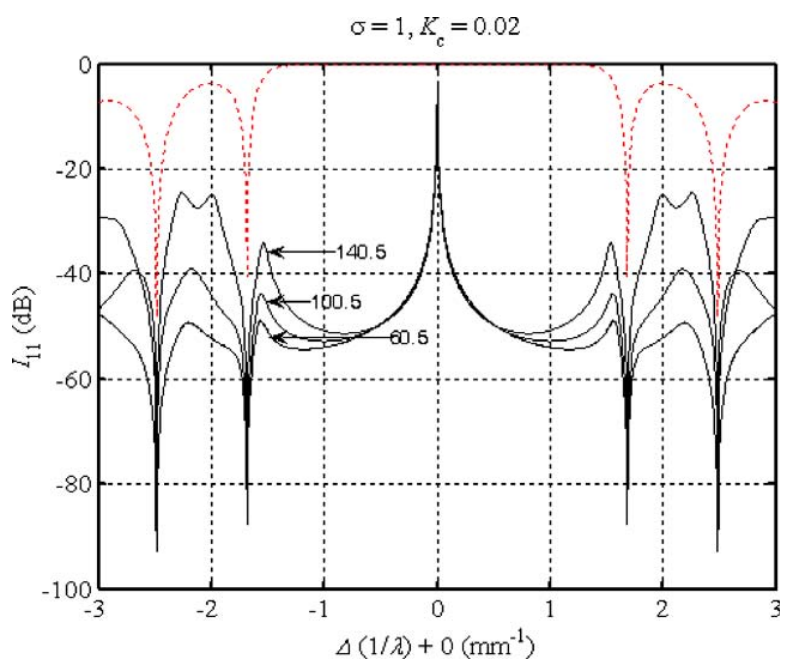

Fig. 4. Reflected intensity as a function of detuning for the type II mirror. The parameter is the mode number of the ring, $n_{3}\left(n_{4}\right)=10$ or 10.5 , while the other parameters are the same as in Fig. 2. In the vicinity of $K=0.5$ the response is virtually independent of $K$. This characteristic also applies to type III mirrors, but with a different set of mode numbers (see Table 1 ).

example in Fig. $2 n_{3} n_{4}$ 10.25, whereas in Fig. $3 n_{3} \quad 10.25$ and $n_{4} 10$, or vice versa. In addition to the significantly larger SS this $\pi$ phase shift also results in a strong dependence of the SS on $K$ as |K $\begin{array}{ll}K & 0.5\end{array} \mid$ approaches zero in Fig. 2, whereas the response in Fig. 3 is virtually insensitive to $K$. Furthermore, as we demonstrate below, the settings of Fig. 2 allow for the shaping of the resonance peak by adjusting $K$. The characteristics of the type II mirror are shown in Fig. 4, indicating significantly larger SS than that displayed in Fig. 3 but similar insensitivity to $K$. The plots of Fig. 4 also apply to type III mirrors using appropriate mode numbers. In all three configurations the location of the bottom coupler, the value of $h$ in Fig. 1, affects only the phase of $S_{11}$.

Figs. 3 and 4 indicate an improvement of the SS with decreasing mode number $N$. This is due to the inverse relationship between mode number and free spectral range (FSR $f_{0} / N$ ) and the atten dant shift of the adjacent resonances into regions with increased grating rejection. If reducing the mode number $N$ to obtain higher
SS is for some reason undesirable, apodized gratings yielding de pressed sidelobes can be used instead of uniform ones.

Observe that the mode numbers are not integers. They must be selected to provide adequate sideband suppression and a (nearly) centered reflection spectrum. The selection rules are determined by the phase shifts within the couplers and the grating arms, and by the imperative for constructive interference at the input port at $f_{0}$. Consider that in a type I mirror the gratings are connected through the ring both clockwise and counter clockwise incurring a phase shift difference of $180^{\circ}$. In a type II mirror the gratings are connected both directly and also through the ring, in which case an extra $180^{\circ}$ phase shift is incurred by crossing the coupler twice. Finally, in a type III mirror the gratings are connected through the ring and through the coupler incurring a $90^{\circ}$ phase dif ference between them. The reflection phases of the gratings, which are subject to their construction, determine the selection rules for $n_{3}$ and $n_{4}$. In our simulations we used the HLHLH sequence, where $H$, the high refractive index was 1.0065 times the low refractive in $\operatorname{dex} L$. These characteristics explain the nature of the mode number selection rules listed in Table 1, where I represent an arbitrary po sitive integer.

The type I mirror, as represented by the spectrum of Fig. 2, dif fers from the others in the effect small variations of $K(\approx 0.5)$ have on the resonant response in the vicinity of $f_{0}$. This is illustrated in Fig. 5, where details of the resonance peaks of Figs. 2 and 3, for four values of $K$ are compared assuming lossless waveguides and a com mon $K_{\mathrm{c}}$. The results show that (a) the high SS type I mirror has stee per rolloff than its moderate SS alternative, and (b) that it displays the typical overcoupling/undercoupling effects of a discontinuity assisted ring [21] near critical coupling: $K_{c, \text { crit }} 2 r /(1+r)$, where $r\left|S_{K, 22}\right|$ is the (2,2) element of (Eq. (1)). In the present example, using $K \quad 0.4944, r\left(f_{0}\right) \quad 0.0112$ and $K_{c, \text { crit }} 0.02215$ which is

Table 1

Mode number selection rules; $I$ is an arbitrary positive integer

\begin{tabular}{llll}
\hline Description & $N$ & $n_{3}$ & $n_{4}$ \\
\hline Type I mirror, very large SS, very sensitive to $K$ & $I+1 / 4$ & $n_{3}=I \pm 1 / 4$ & $n_{3}=I \pm 1 / 4$ \\
& $I-1 / 4$ & $n_{3}=1 / 2 I$ & $n_{4}=1 / 2 I$ \\
Type I mirror, moderate SS, insensitive to $K$ & $I \pm 1 / 4$ & $n_{3}+n_{4}=I \pm 1 / 4$ \\
Type II mirror, high SS, insensitive to $K$ & $I \pm 1 / 2$ & $n_{3}+n_{4}=1 / 2 I$ \\
Type III mirror, high SS, insensitive to $K$ & $I-1 / 4$ & $n_{3,4}=1 / 2 I$ & $n_{43}=I \pm 1 / 4$ \\
\hline
\end{tabular}

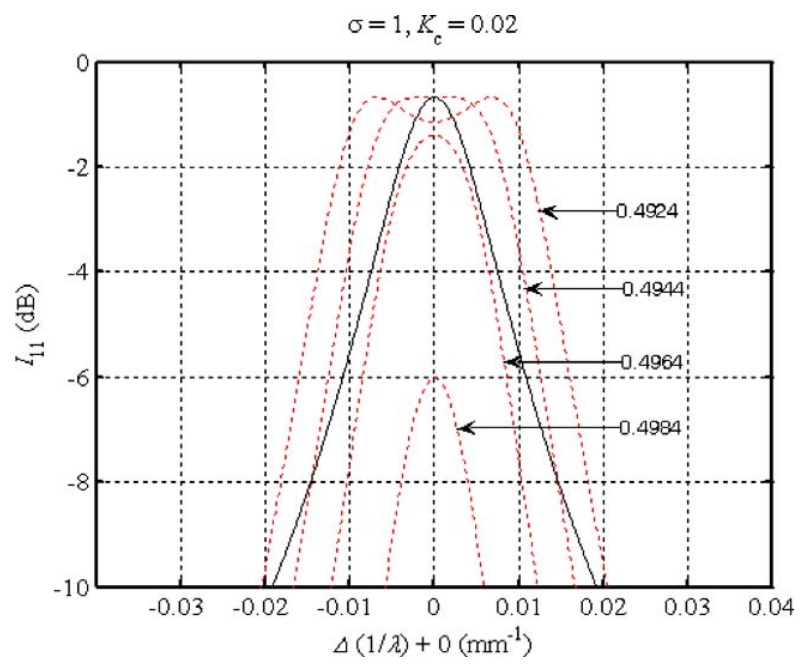

Fig. 5. Passband center of the lossless type I mirror for four values of $K$. Dotted lines: $n_{3}=n_{4}=10.25$, solid line: $n_{3}=10.25, n_{4}=10$ (adjustment in $K$ has no effect on the response). $K_{\mathrm{c}}=0.02, N=60.25, G S=3.92$. 
exactly the value where critical coupling is observed. The effect of varying $K_{\mathrm{c}}$, applied to reflectors using ring resonator mode num bers 60.25 and 140.25 is displayed in Fig. 6, showing the resonance broadening effect of ring diameter reduction, as well as the fact that a change in $N$ does not affect the RL. To illustrate the sensitiv ity of the resonance characteristics the range of $K$ values that provide a reasonably flat (with a maximum of $3 \mathrm{~dB}$ ripple) reso nance peak for the lossless, high SS, type I mirror are summarized in Table 2 for four values of $K_{\mathrm{c}}$.

To compare the RL, the BW and the mode number dependence of the BW, between high SS type I and type II mirrors, we plotted in Fig. 7, using the same $K_{\mathrm{c}}$ and $N$ values as in Fig. 6, the reflected intensity of the type II mirror. Notice that decreasing $N$ from 140 to 60 hardly affects the BW, and that in the type II mirror there is no overcoupling/undercoupling phenomenon. In fact this phe nomenon exists only in the high SS type I mirror on account of the competition between reflected waves re entering the ring from the bottom coupler. A diagram of the reflected intensity in type III mirrors, using $N 140.75$ and $N \quad 60.75$ with $n_{3} 10$, $n_{4} \quad 10.25, \alpha \quad 0$ and GS 3.92 exhibits results that are very simi lar to those of Fig. 7. We shall discuss the sensitivity of $f_{0}$ to incre mental changes in mode numbers in the next Section on tunability.

\section{Tuning sensitivity}

When one of the three mode numbers: $N, n_{3}$ or $n_{4}$ is incremen tally altered the resonance frequency is expected to shift. As we shall see below that this is not always the case. Calculations show that shifts of the central resonance are at least two orders of mag nitude less sensitive to changes in the coupling coefficients than to changes in mode numbers, and that waveguide attenuation has no effect on $f_{0}$. For the high SS type I mirror the evolution of the re

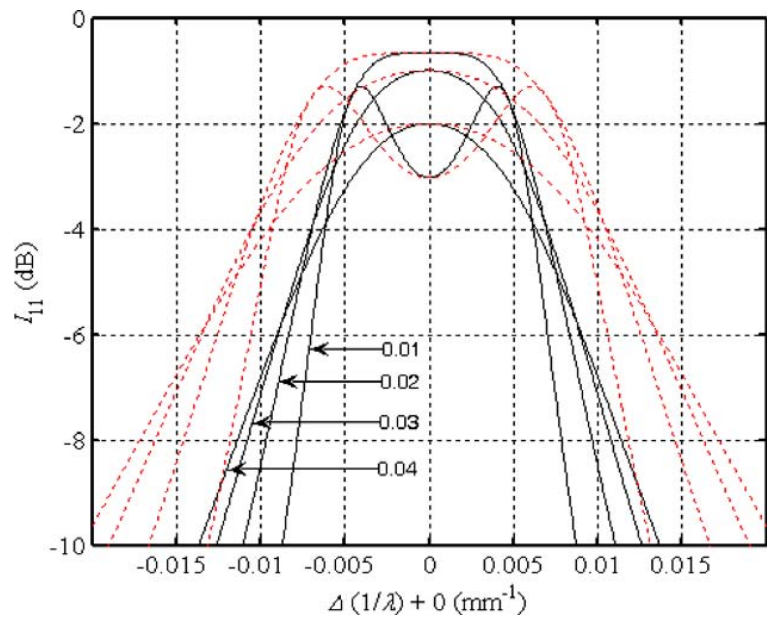

Fig. 6. Resonance peaks of lossless type I mirror. Solid lines: $N=140.25$, dotted lines: $N=60.25, n_{3}=n_{4}=10.25, G S=3.92, K_{c}$ is the parameter (solid and dotted plots with the same extrema correspond to the same $K_{\mathrm{c}}$ ).

Table 2

Useful range of $K$ for lossless, high SS, type I mirror

\begin{tabular}{lll}
\hline$K_{\mathrm{c}}$ & Upper limit of $K$ & Lower limit of $K$ \\
\hline 0.01 & $0.5 \pm 2 \times 10^{3}$ & $0.5 \pm 6 \times 10^{3}$ \\
0.02 & $0.5 \pm 3 \times 10^{3}$ & $0.5 \pm 12 \times 10^{3}$ \\
0.03 & $0.5 \pm 4 \times 10^{3}$ & $0.5 \pm 18 \times 10^{3}$ \\
0.04 & $0.5 \pm 5 \times 10^{3}$ & $0.5 \pm 24 \times 10^{3}$ \\
\hline
\end{tabular}

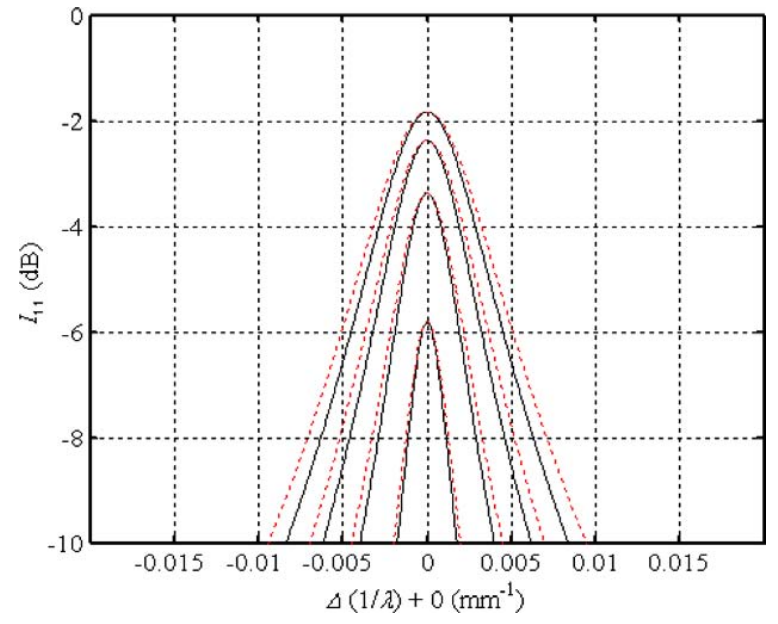

Fig. 7. Resonance peaks of lossless type II mirror. Solid lines: $N=140.5$, dotted lines: $N=60.5, n_{3}, n_{4}=10$ or $10.5, G S=3.92, K_{c}=0.01,0.02,0.03,0.04$, the larger the $K_{\mathrm{c}}$ the smaller the RL and the wider the band. Very similar characteristics apply to type III mirrors with corresponding mode numbers.

flected intensity response as $N$ decreases from 50.25 to 50.0 in increments of 0.05 , covering a frequency span of approximately $300 \mathrm{GHz}$, appears in Fig. 8. This translates to $\delta f \approx 1.2 \mathrm{GHz}$ down shift for $\delta N 1 \times 10^{3}$, very close to the computed value of $1.26 \mathrm{GHz}$. In high SS mirrors the effect of an incremental change in $N$ results simply in a shift of $f_{0}$, while a change in $n\left(n_{3}\right.$ or $\left.n_{4}\right)$ also creates some overcoupling, i.e., affects the shape of the peak.

Morphing of low into high SS state in a lossless type I mirror is demonstrated in Fig. 9, showing the effect of the increase of $n_{3}$ from 10 to 10.25 in increments of 0.05 , while $N$ 50.25, $n_{4} \quad 10.25, K_{c} \quad 0.02$ and $N_{G} \quad 600$ (GS 3.92). Here we observe that incrementation of $n_{3}$ does not shift $f_{0}$.

Like their spectral characteristics, type II and type III mirrors also share similar tuning sensitivities. We found the grating arms nearly six times as sensitive to fractional changes in the optical

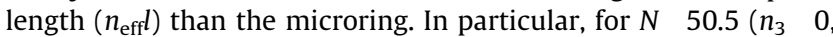
$n_{4} \quad 0$ ) we obtained a downshift of $\delta f 2.22 \mathrm{GHz}$ for $\delta n 1 \times 10^{3}$ or $\delta N 5.9 \times 10^{3}$ (type II mirror), and for $N \quad 50.75\left(n_{3} \quad 0.25\right.$

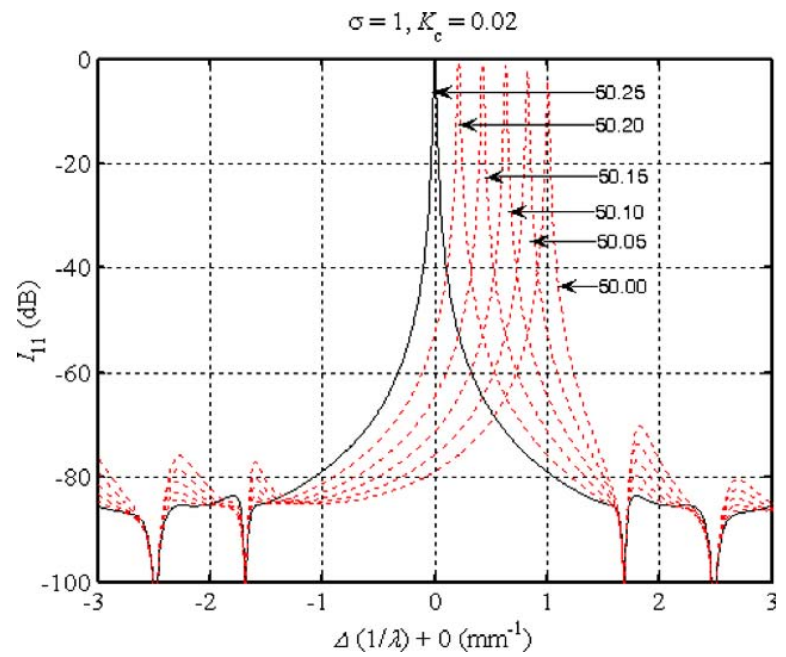

Fig. 8. The evolution of the reflected intensity spectrum in type I mirror. The parameter is $N, n_{3}=n_{4}=10.25, K=0.4944, \alpha=0, N_{G}=600, G S=3.92$. When $N=50$, $\delta f \approx 1.28 \mathrm{GHz}$ for $\delta N=1 \times 10^{3}$. 


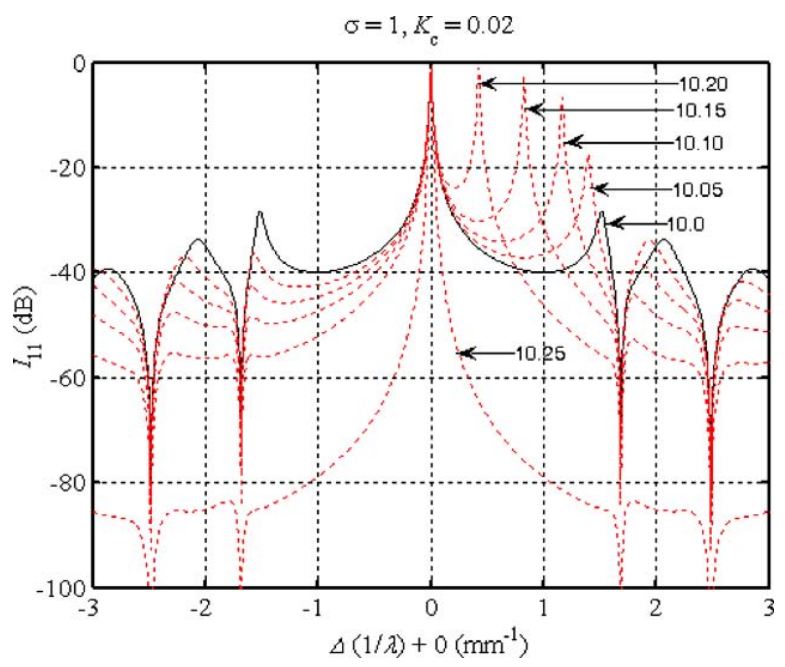

Fig. 9. Type I mirror. Morphing of low into high SS as $n_{3}$ increases from 10 to 10.25 . $N=50.25, n_{4}=10.25, K=0.4944, \alpha=0, N_{G}=600, G S=3.92$. Notice that the resonant peak at $f_{0}$ remains fixed.

$n_{4} \quad 0$ ) a downshift of $\delta f 2.21 \mathrm{GHz}$ resulted for $\delta n 1 \times 10^{3}$ or $\delta N \quad 5.74 \times 10^{3}$ (type III mirror).

\section{Bandwidth and reflection loss}

The BW of the resonant peak at $f_{0}$ is affected by the combined value of the mode numbers $\left(N+n_{3}+n_{4}\right)$ and by the external cou pling coefficient $K_{\mathrm{c}}$. Waveguide attenuation, and/or grating loss through leakage, depresses the peak but does not directly affect the BW. Small changes in $K$ have noticeable effect on the BW only in the high SS type I mirror, through overcoupling or undercou pling, as displayed in Fig. 5.

In general terms, increasing the combined mode number and/or decreasing $K_{\mathrm{c}}$ reduces the BW. Since [22]

$Q \quad \frac{f_{0}}{B W} \quad \frac{2 \pi N}{K_{\mathrm{c}}+2 T_{G}+2 \alpha L} \stackrel{\alpha}{\rightarrow} \frac{2 \pi N}{K_{\mathrm{c}}+2 T_{G}}$

where $T_{G}$ denotes the power loss through a grating, these results meet expectations. A decrease in the value of $K_{\mathrm{c}}$ also reduces the peak value of the resonance, because with reduced coupling less external power is needed to maintain cavity oscillation. Some of these effects are displayed below. Fig. 10 shows that the BW of type II (as well as type III) mirrors are significantly less sensitive to $K_{c}$ than that of the type I configuration. Note that the reflection coef ficients of the gratings of the type II (type III) mirrors have been adjusted to provide the same band center loss as the type I mirror. Fig. 11 plots the resonance peak in type I and type II (type III) mirrors for three nominal mode numbers using $K_{c} \quad 0.02$. Here we observe that the BW spread in the latter configurations is once again significantly smaller than that in the former.

Reflection loss, defined as the level of the reflected intensity rel ative to the incident intensity, i.e., $\left|S_{11}\right|^{2}$, depends on the leakage through the gratings determined by the GS, the resonator loss and the power escaping through port 2, i.e., $\left|S_{21}\right|^{2}$. Power conserva tion requires that the sum of these four components equals the input power entering port 1 . Resonator loss is determined by the per turn power loss $2 \alpha L$, but reaches a peak at $f_{0}$ due to resonant enhancement. Transmission loss also peaks at $f_{0}$ [22]. If there is no leakage through the gratings due to a very large GS, the RL is a function of $\alpha, L$ and $K_{\text {c. }}$. In contrast, when waveguide loss is negligible and the RL is driven by leakage through the gratings,

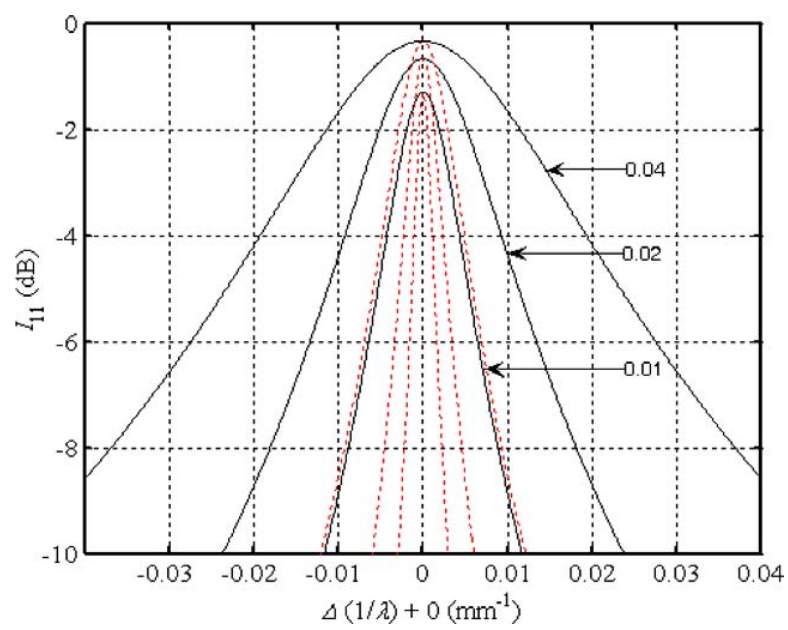

Fig. 10. The effect of $K_{\mathrm{c}}$ on BW. Solid lines: moderate SS type I mirror, $N=50.25$, $n_{3}=0, n_{4}=0.25, N_{G}=600, \alpha=0$, dash-dot lines: type II mirror, $N=50.5, n_{3}=0, n_{4}=0$, $N_{G}=736, \alpha=0$, (curves having the same maxima belong to the same $K_{c}$ ). Characteristics for the type III mirror are virtually identical to those of the type II mirror.

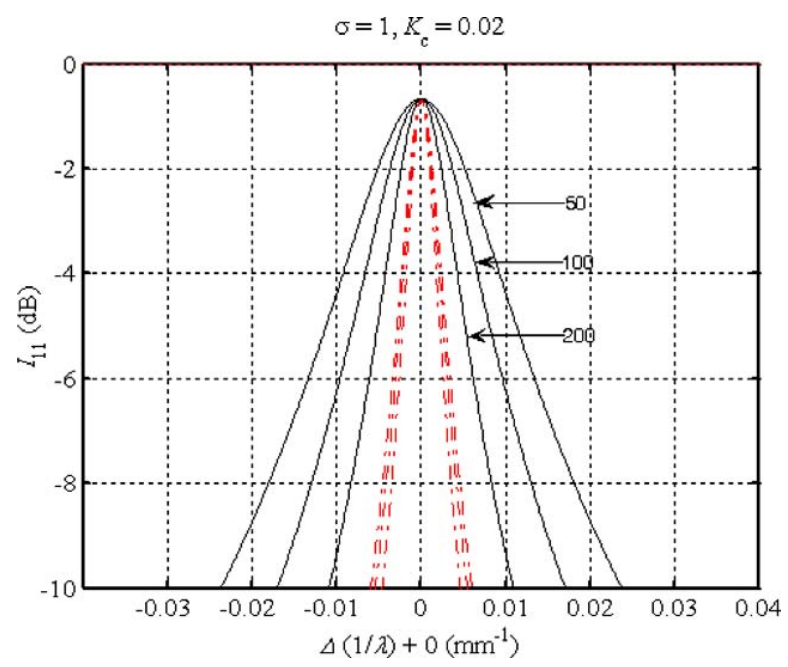

Fig. 11. The effect of $N$ on BW. Solid lines: moderate SS type I mirror, $n_{3}=0$, $n_{4}=0.25, N_{G}=600$, dash-dot lines: type II mirror, $n_{3}=0, n_{4}=0, N_{G}=736, K_{\mathrm{c}}=0.02$, $\alpha=0$. The parameter is the nominal mode number, the smaller the $N$ the wider is the BW. Type II and type III mirror responses are virtually identical.

only $K_{\mathrm{c}}$ and the GS affect RL. In any event, loss within the ring and leakage through the gratings reach a maximum while output at port 2 dips to a minimum at resonance. Fig. 12 plots for type I and for type II mirrors the RL as a function of $\alpha$ for three values of $K_{\mathrm{c}}$ when there is no leakage through the gratings $\left(N_{G} 2000\right.$, GS 13), $K \quad 0.5$ and the nominal mode numbers are $N \quad 100$, and $n_{3} \quad n_{4} \quad$ 0, respectively. Similarly, Fig. 13 is a $R L \quad R L(G S)$ plot for type I and type II mirrors of the same description when $\alpha 0$. The responses of type III mirrors are not shown because they are very similar to those of the corresponding type II mirrors. The dia grams show that type I mirrors have significantly smaller RL than the other types, and that increasing $K_{\mathrm{c}}$ reduces RL. (Note that we use a positive number for loss, a negative level.)

Referring to Figs. 12 and 13 we note that identical $\alpha L$ products do not ensure identical RL at $f_{0}$, however the RL is independent of $N$ when it is driven by leakage loss $\left(\begin{array}{ll}\alpha & 0\end{array}\right)$. Also, the output level at port 2, i.e., $\left|S_{21}\right|^{2}$ at resonance rises with rising resonator loss 


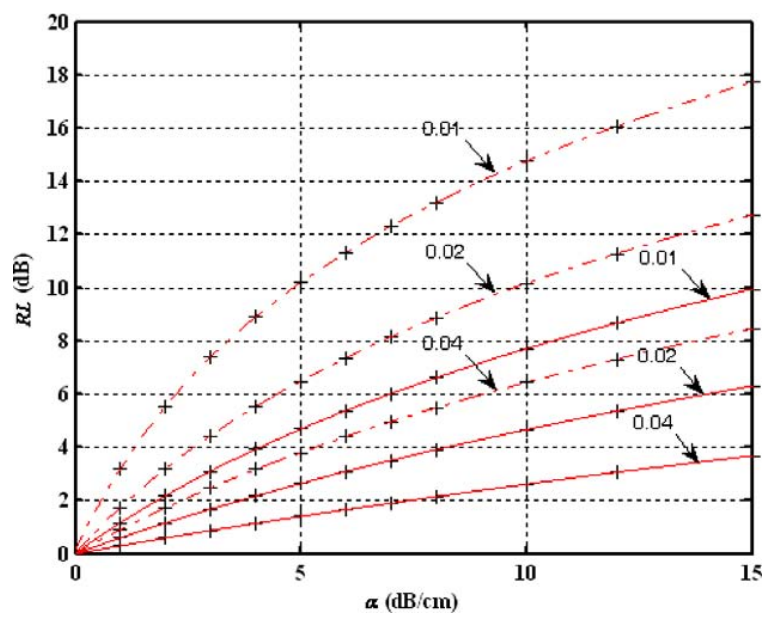

Fig. 12. Computed reflection loss at $f_{0}$ in type I (solid lines) and type II (dash-dot lines) mirrors as a function of $\alpha$. The nominal mode numbers are: $N=100$, $n_{3}=n_{4}=0, G S=13, K=0.5$. The parameter is $K_{\mathrm{c}}$. The properties of the type III mirror are the same as those of the type II mirror.

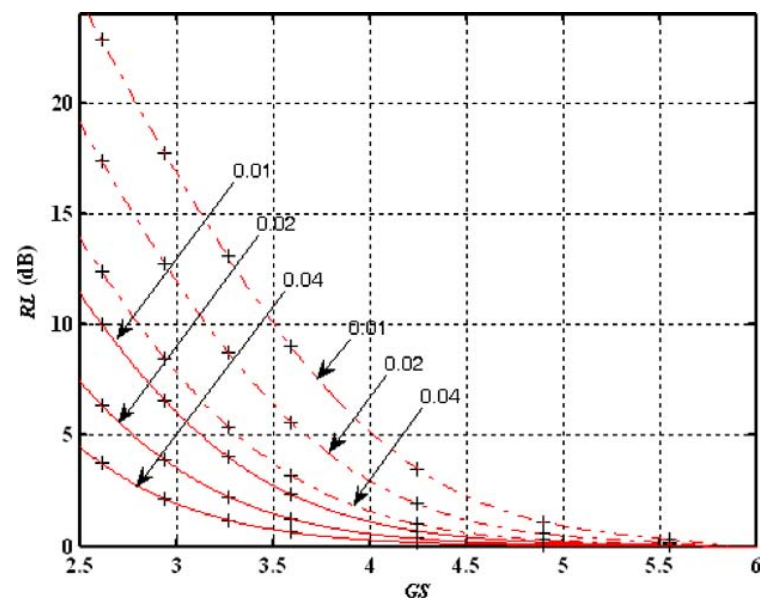

Fig. 13. Computed reflection loss at $f_{0}$ in type I (solid lines) and type II (dash-dot lines) mirrors as a function of the GS. The nominal mode numbers are: $N=100$, $n_{3}=n_{4}=0, \alpha=0, K=0.5$, the parameter is $K_{\mathrm{c}}$. The characteristics of the type III mirror are the same as those of the type II mirror.

and/or rising leakage through the gratings, a phenomenon which at first glance seems counterintuitive and indicates a partition be tween the reflected power on the one hand and the absorbed and transmitted power on the other. Finally, we note that for the same level of internal or leakage loss, the transmitted power levels at port 2 are always (significantly) higher in type II and type III mir rors than for the type I mirror.

\section{Group delay in reflection}

We also computed the group delay in reflection: $\tau_{11}$, for lossless and moderately lossy reflectors, for several coupling coefficients $K_{\mathrm{c}}$. The group delay was computed from (Eq. (5)) using the expression [22]

$$
\tau_{11} \quad \operatorname{Im}\left[\frac{1}{S_{11}} \frac{\partial S_{11}}{\partial \omega}\right]
$$

The group delay in reflection is plotted in Fig. 14, over the cen tral region of the passband, for high and moderate SS type I mirrors for several values of $K$. The dotted curves show the effect of slight

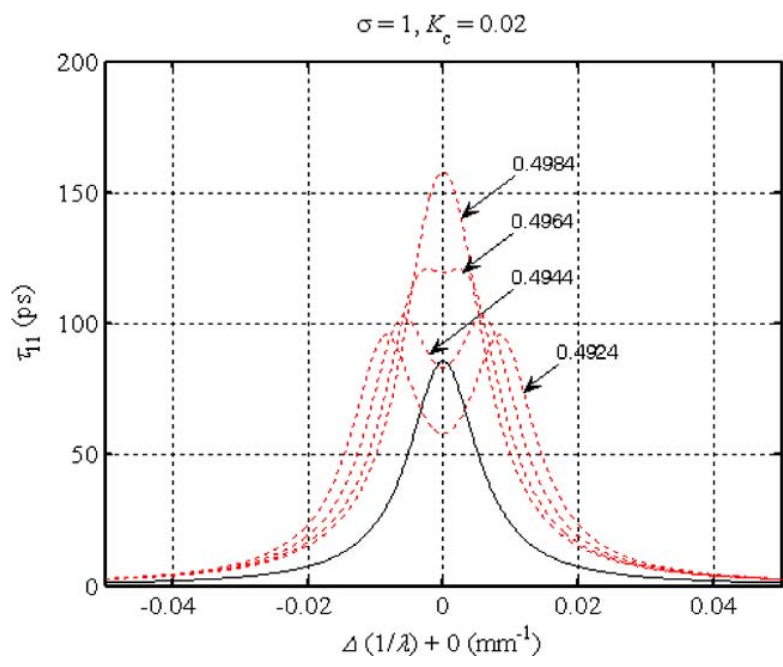

Fig. 14. Group delay in reflection of type I mirrors, $\alpha=0, N=100.25, N_{G}=600$ (GS $=3.92$ ), $K_{\mathrm{C}}=0.02$, the parameter is $K$. Dotted lines: $n_{3}=n_{4}=0.25$ (high SS), solid line: $n_{3}=0.25, n_{4}=0$ (same plot for all four $K$ values).

changes in $K$ in the high SS configuration, while the single solid line shows that adjustment of $K$, while $K \approx 0.5$, has no effect on $\tau_{11}$ in the moderate SS case. Notice that the coefficient $K \quad 0.4944$, found to provide critical coupling for $I_{11}$ at $K_{\mathrm{c}} \quad 0.02$ in the high SS type I mirror, does not provide flat top $\tau_{11}$ characteristic. Instead, flat top $\tau_{11}$ characteristic is obtained for $K \quad 0.4964$. Fig. 15 plots $\tau_{11}$ for high SS (dotted lines) and moderate SS (solid lines) type I mirrors, using $K \quad 0.4944$, for three microring mode numbers. Both Figs. 14 and 15 were computed using $\alpha \quad 0, K_{\mathrm{c}} \quad 0.02, N_{G} 600$ (GS 3.92) and vanishing grating arm lengths. Group delay in reflection in type II and type III mirrors show the same single peak characteris tic as those of the solid lines in Figs. 14 and 15. Peak values of $\tau_{11}$ in ps, obtained assuming $\alpha \quad 0$ and $4 \mathrm{~dB} / \mathrm{cm}, N_{G} \quad 600$ (GS 3.92 ) and $K \quad 0.5$ are listed in Table 3 for moderate SS type I and for type II mirrors indicating significantly larger group delays for the latter. Corresponding values for type III mirrors $\left(\begin{array}{llll}n_{3} & 0, n_{4} & 0.25\end{array}\right)$ were found to be very close to those of type II mirrors. Since, if used as a laser mirror, $\tau_{11}$ contributes to its photon lifetime, a larger

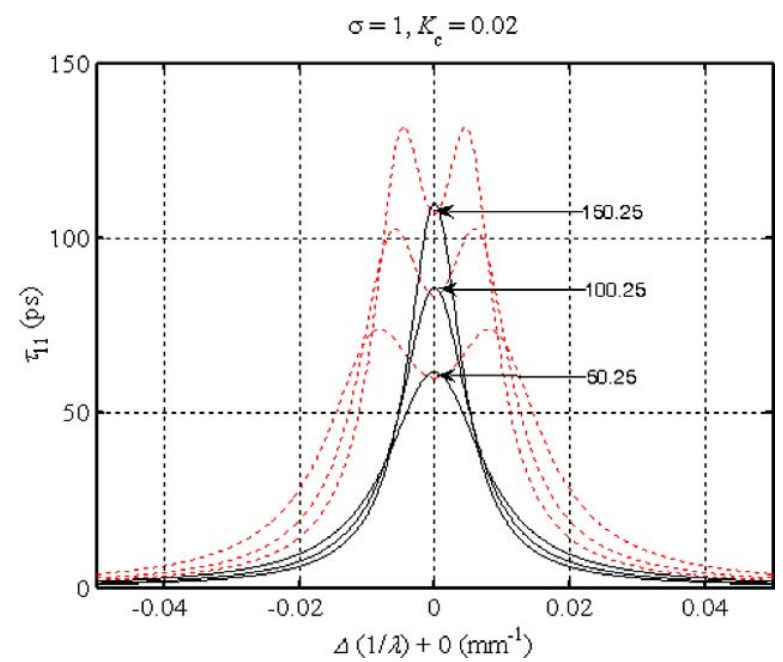

Fig. 15. Group delay in reflection of lossless type I mirrors. Solid curves: moderate SS $\left(n_{3}=0.25, n_{4}=0\right)$, dotted curves: high SS $\left(n_{3}=n_{4}=0.25\right)$. The parameter is $N$, $N_{G}=600(G S=3.92), K_{\mathrm{c}}=0.02, K=0.4944$. 
Table 3

Peak group delay in reflection in ps

\begin{tabular}{llccr}
\hline Nominal $N$ & $\alpha(\mathrm{dB} / \mathrm{cm})$ & $K_{\mathrm{c}}=0.01$ & $K_{\mathrm{c}}=0.02$ & $K_{\mathrm{c}}=0.04$ \\
\hline 50 & 0 & 115.3 & 61.7 & 31.7 \\
& & 269.8 & 177.5 & 104.6 \\
& 4 & 85.2 & 51.9 & 28.9 \\
100 & & 147.8 & 115.0 & 79.3 \\
& 0 & 160.4 & 85.8 & 44.1 \\
& 4 & 296.8 & 195.2 & 115.1 \\
& 4 & 107.6 & 68.0 & 38.9 \\
& & 155.6 & 122.2 & 85.1
\end{tabular}

$N_{G}=600, G S=3.92, K=0.5$. Top figures: moderate SS type I mirror $\left(n_{3}=0, n_{4}=0.25\right)$, bottom figures: type II mirror $\left(n_{3}=n_{4}=0\right)$. Delays in type II and III mirrors are the same.

group delay in reflection raises the $Q$ and narrows the line width. As can be gleaned from Table 3, whether it is caused by waveguide attenuation or by leakage through the gratings, loss flattens the $\tau_{11}$ characteristics and reduces its peak.

\section{Conclusions}

Three grating assisted ring resonator based narrow band opti cal reflectors have been analytically and numerically examined. The grating pair is instrumental in enabling a single microring to be used as a mirror free of a repetitive spectrum. Reflection and group delay characteristics have been presented and the effects of waveguide loss and leakage through finite length gratings eval uated. Tuning sensitivity in response of optical length $\left(n_{\text {eff }} L\right)$ varia tions in both the microring and the grating arms was investigated. We also examined shaping the peak of the resonance characteristic of the high SS type I mirror and found it feasible at a cost of the technologically challenging fine adjustment of $K$. Among the mirror configurations examined significant differences have been found in
$\mathrm{SS}$, in BW, in RL, in group delay, and in the sensitivity to deviations in $K$. These differences and the potential radiation loss associated with small bending radii used in one design are expected to guide the designer.

\section{Acknowledgments}

The support of the Spanish Ministry of Education project CICYT TEC2006 13273 C03 03/MIC and of the Natural Sciences and Engi neering Research Council of Canada are acknowledged.

\section{References}

[1] B. Liu, A. Shakouri, J.E. Bowers, IEEE Photon. Technol. Lett. 14 (2002) 600.

[2] J.K.S. Poon, J. Scheuer, A. Yariv, IEEE Photon. Technol. Lett. 16 (2004) 1331

[3] I. Chremmos, N. Uzunoglu, IEEE Photon. Technol. Lett. 17 (2005) 2110.

[4] Y. Chung, D.-G. Kim, N. Dagli, IEEE Photon. Technol. Lett. 17 (2005) 1773.

[5] .G.T. Paloczi, J. Scheuer, A. Yariv, IEEE Photon. Technol. Lett. 17 (2005) 390.

[6] O. Schwelb, J. Lightwave Technol. 23 (2005) 3931.

[7] C. Vázquez, S. Vargas, J.M.S. Peña, Appl. Opt. 39 (2000) 1934

[8] C. Vázquez, S. Vargas, J.M.S. Pena, P. Corredera, IEEE Photon. Technol. Lett. 15 (2003) 1085 .

[9] C. Vázquez, J. Montalvo, P.C. Lallana, J.M.S. Pena, Proc. SPIE 5840 (2005) 315.

[10] C. Vázquez, S. Vargas, J.M.S. Pena, J. Lightwave Technol. 23 (2005) 2555.

[11] O. Schwelb, IEEE Trans. Microwave Theory Tech. 46 (1998) 1409.

[12] G. Lenz, B.J. Eggleton, C.K. Madsen, R.E. Slusher, IEEE J. Quantum Electron. 37 (2001) 525.

[13] B. Vizoso, C. Vázquez, R. Civera, M. López-Amo, M.A. Muriel, J. Lightwave Technol. 12 (1994) 294.

[14] D.G. Rabus, M. Hamacher, U. Troppenz, H. Heidrich, IEEE Photon. Technol. Lett. 14 (2002) 1442.

[15] O. Schwelb, I. Frigyes, Microwave Opt. Technol. Lett. 42 (2004) 427.

[16] M.W. Pruessner, T.H. Stievater, M.S. Ferraro, W.S. Rabinovich, Opt. Express 15 (2007) 7557.

[17] A.V. Tsarev, F. De Leonardis, V.M.N. Passaro, Opt. Express 16 (2008) 3101.

[18] O. Schwelb, Opt. Commun. 281 (2008) 1065.

[19] H.M. Stoll, IEEE Trans. Circ. Syst. CAS-26 (1979) 1065.

[20] S. Xiao, M.H. Khan, H. Shen, M. Qi, J. Lightwave Technol. 26 (2008) 228.

[21] O. Schwelb, I. Frigyes, J. Lightwave Technol. 19 (2001) 380.

[22] O. Schwelb, J. Lightwave Technol. 22 (2004) 1380. 